\title{
Central venous catheter-related infections in patients receiving short-term hemodialysis therapy: incidence, associated factors, and microbiological aspects
}

\author{
Mayra Gonçalves Menegueti ${ }^{[1]}$, Natália Cristina Betoni ${ }^{[2]}$, \\ Fernando Bellissimo-Rodrigues ${ }^{[3]}$ and Elen Almeida Romão ${ }^{[2]}$
}

[1]. Comissão de Controle de Infecção Hospitalar, Unidade de Transplante Renal, Faculdade de Medicina de Ribeirão Preto, Universidade de São Paulo, Ribeirão Preto, SP, Brasil. [2]. Unidade de Transplante Renal, Divisão de Nefrologia, Faculdade de Medicina de Ribeirão Preto, Universidade de São Paulo, Ribeirão Preto, SP, Brasil. [3]. Divisão de Epidemiologia, Departamento de Medicina Social, Faculdade de Medicina de Ribeirão Preto, Universidade de São Paulo, Ribeirão Preto, SP, Brasil.

\begin{abstract}
Introduction: Bloodstream infections are the second most common cause of death among patients on hemodialysis. This study aimed to evaluate the incidence of and risk factors associated with central venous catheter-related infections in patients undergoing hemodialysis, and to identify and characterize the type and antimicrobial susceptibility profiles of the primary microorganisms isolated during one year of follow-up. Methods: A prospective cohort study was conducted in 2014 in a hemodialysis referral center. We included 200 outpatients with acute kidney injury who had no permanent venous access. A nurse assessed the patients for signs of infection three times per week during dressing changes. The clinicopathologic characteristics of patients with and without local or systemic infection were compared. Results: Fifty-five episodes of catheter-related infections occurred in 43 (22\%) patients; 38 (69\%) were bloodstream infections and 17 (31\%) were local infections. Thirty-two (75\%) patients with infection had femoral vein catheter placement. In total, 6,240 hemodialysis sessions were performed; the rates of primary bloodstream and local infection were 6.1 and 2.7 episodes per 1,000 patients on daily dialysis, respectively. In the univariate analysis, diabetes was significantly associated with the development of infection, while level of education, ethnicity, age, and sex were not. Gram-negative bacteria were primarily isolated from blood culture specimens ( $55 \%$ of samples). Of the Gram-negative isolates, $56 \%$ were resistant to the carbapenems. Conclusions: We identified a high incidence of catheter-related infections caused by resistant microorganisms in patients undergoing hemodialysis via central venous catheters.
\end{abstract}

Keywords: Bloodstream infection. Hemodialysis. Resistant microorganisms.

\section{INTRODUCTION}

In Brazil, the estimated number of patients with end-stage renal disease and receiving renal replacement therapy increased from 42,695 in 2000 to 112,004 in $2014^{1}$. All-cause mortality is 6.5-7.9 times greater in patients on dialysis treatment than it is in the general population ${ }^{1}$. Among elderly patients, mortality due to infection is two times higher among those on dialysis than among those not on dialysis ${ }^{2}$. Bloodstream infection (BSI) is the leading cause of hospitalization and the second most common cause of death among patients on hemodialysis ${ }^{3,4}$.

Various studies have shown that the use of native arteriovenous fistulas for hemodialysis is superior to the use of central venous catheters, as the former is associated with fewer mechanical and infectious complications and is less costly $y^{5-8}$.

Corresponding author: Mayra Gonçalves Menegueti.

e-mail: mayramenegueti@usp.br

Received 4 November 2016

Accepted 17 November 2017
However, in Brazil, it is estimated that $16 \%$ of patients on hemodialysis use central venous catheters for short- or long-term dialysis ${ }^{1}$. In addition, patients on hemodialysis have impaired defense mechanisms related to their underlying comorbidities (such as diabetes mellitus) and the malnutrition that results from uremia and dialysis treatment ${ }^{6}$.

The causes of these infections and preventive measures for these complications have been widely discussed. Colonization of the skin around the insertion site, of the catheter tip, in the catheter by hematogenous dissemination from another location, and contamination of the locking solution are some of the factors causing these events. Other factors include contamination at the time of catheter insertion due to inadequate aseptic measures and iatrogenic contamination during any subsequent manipulation of the catheter?.

Given the high reported incidence of infectious complications in patients on hemodialysis, as well as the morbidity and mortality related to these events, the present study aimed to evaluate the incidence of and risk factors associated with catheter-related infections in patients with acute kidney injury 
undergoing dialysis, and to identify and characterize the antimicrobial susceptibility profiles of primary microorganisms isolated during a 1-year follow-up period.

\section{METHODS}

\section{Ethical considerations}

This was a prospective cohort study conducted in a high-complexity hospital in the State of São Paulo, Brazil, during 2014. The study was approved by the Research Ethics Committee of the University Center Hermínio Ometto, number 38152314.3.0000.5385. Written informed consent was provided by each patient or his/her legal guardian.

The study included 200 patients with acute kidney injury who required hemodialysis but did not have permanent venous access. All central venous catheters were inserted by nephrologists according to the procedure described in the institutional protocol that includes, in the following order: I) hand washing; II) complete gowning (cap, mask, gown, gloves, and protective glasses); and III) antisepsis of the insertion site and antisepsis of the expanded field using chlorhexidine and alcohol, respectively. Sites of insertion, in order of preference, were the internal jugular vein then the femoral vein. To avoid causing possible subclavian vein stenosis, the subclavian vein was not used. All catheters were for short-term use, were sutured to the skin, and were covered with a bandage using sterile gauze and adhesive tape. The connections were occluded with sterile gauze.

The catheters were used exclusively for hemodialysis. After each hemodialysis session, the catheters were filled with a solution of sodium heparin without using any antimicrobial solution. Antiseptic dressings were not used. Dressing changes were aseptically performed before each session, three times per week. A nurse assessed the insertion site for signs of infection when changing the dressing. If an infection was suspected, the nephrologist was summoned. In cases of local erythema only, the catheter position was maintained, provided that other clinical evidence of BSI was excluded. If purulent secretions were noted or an infection was suspected, two blood samples were collected, the catheter was removed aseptically, and its tip was sent for microbiologic culture. Blood samples $(20 \mathrm{~mL})$ were collected in Bactec Becton Dikinson, Brazil containers and the cultures were processed using the Vitek ${ }^{\mathrm{TM}}-2$ (bioMérieux, Brazil) automated system. The samples were incubated for 5-7 days. To identify catheter tip contamination, approximately $50 \mathrm{~mm}$ of the catheter tip was passed through pre-made blood agar plates, in accordance with the semi-quantitative method. Catheters whose cultures grew $>15$ colony-forming units on the corresponding blood agar plates were considered to be colonized.

At the time of inclusion in the study, clinical and demographic data were collected from the patients. The patients were followed during the entire period of hemodialysis, until they had a functional arteriovenous fistula created or were referred to another service. Catheters were removed in the following situations: malfunction, presence of erythema with or without purulent secretions, bacterial infection, or fever without another probable source of infection. Infections were defined according to the following criteria established by the Centers for Disease Control and Prevention (CDC) ${ }^{10}: 1$ ) Local catheter infection: This was defined as the presence of one or more of the following signs or symptoms with no other known cause: Pain, erythema, warmth, exudate or purulent secretion, and growth of microorganisms in cultures of the catheter tips. 2) Primary catheter-related BSI with laboratory confirmation (PILC): PILC was defined as presence of at least one positive blood culture, collected from a peripheral vein while the catheter was in place, or two positive samples when the skin was contaminated with microorganisms, together with at least one of the following symptoms: Fever $\left(>38^{\circ} \mathrm{C}\right)$, shivering, or hypotension. 3) Primary catheter-related BSI without laboratory confirmation (PIWLC): PIWLC was defined based on the aforementioned PILC criteria but with negative blood cultures results.

In all cases, clinical, radiological, laboratory and microbiological data were evaluated to confirm the absence of any another probable source of infection.

All collected data were entered into a Microsoft Excel ${ }^{\circledR}$ spreadsheet and subsequently analyzed using SPSS 22.0 software. In the univariate analysis, Student's $t$-test or the MannWhitney $U$-test was used to compare continuous variables that followed a normal and non-normal distribution, respectively, according to the Kolmogorov test. Categorical variables were evaluated using the chi-square test or Fisher's exact test, as appropriate. For all analyses, an $\alpha$-value of $5 \%$ was established; hence, associations with a $p$-value $<0.05$ were considered statistically significant.

\section{RESULTS}

The demographic data of the 200 patients evaluated in this study are shown in Table 1. Of these, serologic testing revealed that $14(7 \%)$ patients were infected with human immunodeficiency virus, eight (4\%) had hepatitis $\mathrm{C}$ infection, and two (1\%) had hepatitis B infection. The presence of antibodies against hepatitis B surface antigen was assessed in 158 patients; the results were positive in 93 (58.9\%). Ninetyeight $(49 \%)$ patients died during the follow-up period; $12(6 \%)$ died due to infection-related causes.

A total of 55 catheter-related infectious episodes occurred in 43 (22\%) patients: Five patients had two episodes of PILC each, two had three episodes of PILC each, and two had two episodes of local catheter infection each. Of the 55 episodes of infection, $38(69 \%)$ were catheter-related BSIs and $17(31 \%)$ were local infections (Table 1). Overall, 36 (65\%) episodes of infection were confirmed by laboratory findings. Of the 43 patients who had a catheter-related infection, 32 (75\%) had venous catheters placed in the femoral vein.

During the study period, 6,240 hemodialysis sessions were performed. The incidence of primary catheter-related BSI was 6.1 episodes per 1,000 hemodialysis sessions. The incidence of local catheter site infection was 2.7 episodes of per 1,000 hemodialysis sessions. Univariate analysis revealed that diabetes mellitus as a comorbid condition was the only variable associated with the development of catheter-related infections. 
TABLE 1: Univariate analysis of demographic and clinical characteristics of patients on hemodialysis with and without catheter infections during one year of follow-up (Ribeirão Preto, São Paulo State, Brazil, 2014).

\begin{tabular}{|c|c|c|c|}
\hline & With infection & Without infection & $p$-value \\
\hline Patients, n (\%) & $43(22.0)$ & $157(78.0)$ & $\mathrm{n} / \mathrm{a}$ \\
\hline Male sex, n (\%) & $24(56.0)$ & $81(52.0)$ & 0.37 \\
\hline \multicolumn{4}{|l|}{ Level of education, $n(\%)$} \\
\hline primary school incomplete & $14(33.0)$ & $49(31.0)$ & 0.71 \\
\hline high school diploma & $4(9.0)$ & $22(14.0)$ & 0.63 \\
\hline university complete & $2(4.0)$ & $5(3.0)$ & 0.93 \\
\hline \multicolumn{4}{|l|}{ Race, n (\%) } \\
\hline white & $32(74.0)$ & $116(73.0)$ & 0.79 \\
\hline HIV & $3(7.0)$ & $11(7.0)$ & $>0.99$ \\
\hline $\mathrm{HCV}$ & $4(9.5 .0)$ & $4(2.5 .0)$ & 0.61 \\
\hline Diabetes mellitus & $146(73.0)$ & $54(27.0)$ & 0.0001 \\
\hline
\end{tabular}

SD: standard deviation; $\mathrm{n} / \mathrm{a}$ : not applicable; HIV: human immunodeficiency virus; HCV: hepatitis C virus.

The microorganisms identified and their antimicrobial susceptibility profiles are shown in Table 2. Of the 36 episodes of PILC, $20(56 \%)$ occurred in patients with femoral vein catheters and $16(44 \%)$ in patients with internal jugular vein catheters. Gram-negative microorganisms were the most common, being detected in $50 \%$ of the blood samples from 10 patients with femoral vein catheters and 8 patients with internal jugular vein catheters. Of the gram-negative organisms, $10(56 \%)$ were resistant to the carbapenems and one (5\%) was resistant to polymyxin $\mathrm{B}$. The gram-positive microorganisms were the second most prevalent group, with Staphylococcus aureus being the most common (36\%). Table 2 also shows the microorganisms identified in the catheter tips of the 17 cases of local catheter infection; gram-positive microorganisms were the most common [65\% $(n=11)]$ and $41 \%$ of specimens cultured Staphylococcus spp.

\section{DISCUSSION}

In this study, we included patients with central venous catheters inserted for the sole purpose of providing hemodialysis over a short-term period. During the follow-up period, $22 \%$ of these patients had at least one episode of catheter-related infection. However, no cases of endocarditis or abscess were identified. In a study of 210 patients with chronic kidney disease undergoing long-term hemodialysis, $31 \%$ developed bacteremia: $17.1 \%$ had endocarditis, $5.7 \%$ developed abscesses, and $4.8 \%$ developed sepsis ${ }^{11}$. Infectious complications associated with hemodialysis performed via temporary catheters represent one of the most important causes of morbidity and mortality among patients with renal insufficiency ${ }^{12,13}$.
A prospective multicenter study identified that an infectious etiology was responsible for $23 \%$ of all deaths among 1,846 patients on long-term hemodialysis, while the annual rate of infection was $35 \%{ }^{14}$. In this study, although the mortality rate during the period of observation was relatively high (49\%), the cause of death was infection-related in only $6 \%$ of patients. The higher observed mortality rate can be explained by the fact that the study population included elderly patients, patients with multiple comorbidities, and was restricted to patients with acute kidney injury.

A study conducted in a university hospital that stratified infection rates by type of vascular access (following the CDC criteria) reported infection rates of $0.9,2.39$, and 5.5 episodes per 1,000 hemodialysis sessions among patients with arteriovenous fistulas, long-term placement of central venous catheters, and short-term placement of central venous catheters, respectively ${ }^{15}$. However, a systematic review of studies published during the period 1990-2008 observed that local and systemic catheterrelated infection rates ranged from 19.92 to 29.2 episodes per 1,000 hemodialysis sessions. In the present study, we observed an average of 8.8 catheter-related infection episodes (local and systemic), a result well above that reported by Gnass et a ${ }^{15}$, but well below that reported in the studies cited in the review. This difference may be due to factors including the notification criteria used, institutional protocols regarding the care and maintenance of indwelling venous catheters, patients' adherence to guidelines, and the profile of patients receiving hemodialysis. Thus, we emphasize the importance of disseminating epidemiologic data regarding the occurrence of these infections in patients 
TABLE 2: Microorganisms identified in blood cultures and catheter tips collected from patients with laboratory confirmed bloodstream or local catheter infections during one year of follow-up (Ribeirão Preto, São Paulo, Brazil, 2014).

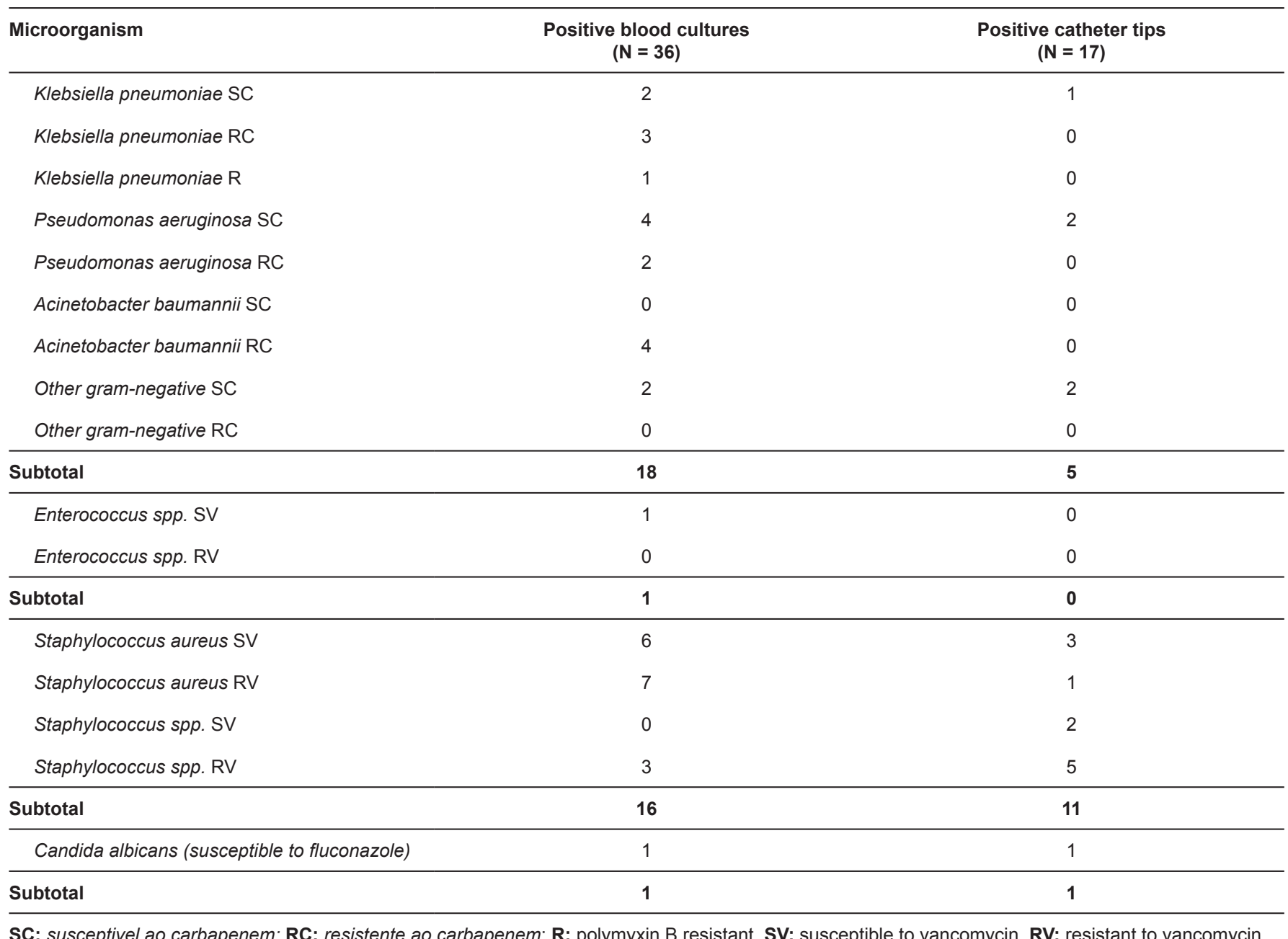

receiving different types of hemodialysis and in patients with acute and chronic kidney disease, according to standardized criteria defining catheter-related infections.

Regarding the profiles of the isolated microorganisms, we noted a difference between the types isolated from local and systemic infections, with a predominance of Gram-negative bacteria in systemic infections and of gram-positive bacteria in local infections. A study ${ }^{16}$ that aimed to identify the incidence of infection and evaluate the profile of microorganisms isolated showed that $35 \%$ of bacteremic episodes were caused by gramnegative bacteria, a rate consistent with those reported in other studies $^{17-19}$. Other study that use the medical records of 239 patients undergoing hemodialysis at the Department of Nephrology of the University Hospital of Heraklion, Crete, Greece, identify that, 3 out of 8 isolated (37.5\%) Enterobacter spp. strains, 4 out of $5(80 \%)$ Acinetobacter spp. strains, and 2 out of 7 (29\%) Klebsiella spp. strains were sensitive only to colistin ${ }^{19}$.

The occurrence of infections caused by antimicrobialresistant microorganisms is a global public health problem. Resistant bacteria such as Acinetobacter baumannii,
Staphylococcus aureus, Pseudomonas aeruginosa, Klebsiella pneumoniae, and Enterococcus spp., are commonly found in healthcare institutions ${ }^{20}$. In another cross-sectional study, conducted in the University Hospital of the Federal University of São Paulo, from 156 patient in hemodialysis who used central venous catheters identify that all $S$. aureus strains were found to be fully susceptible to teicoplanin and vancomycin, $P$. aeruginosa was fully sensitive to clavulanic acid and tazobactam, and $80 \%$ of A. baumannii samples were susceptible to imipenem ${ }^{21}$. In contrast, in the current study we found that the gram-negative bacteria displayed greater resistance to antimicrobial agents, with $56 \%$ being resistant to the carbapenems. This might be because the patients had acute kidney injury, multiple comorbidities, and required hospitalization, which in turn could have modified the microflora profile of these patients, thereby facilitating colonization or infection with multidrug-resistant organisms.

In the present study, the occurrence of catheter-related infection was higher in patients with than without diabetes. A case-control study conducted in Greece over a 7-year period 
evaluated 102 patients with chronic kidney disease (cases) and 137 controls; the multivariate logistic regression model identified the presence of diabetes mellitus [odds ratio (OR): 2.651; $p<0.001$ ], absence of an arteriovenous fistula (OR: 2.933; $p=0.047)$, low serum albumin concentration (OR: 1.976; $p=0.040$ ), and low hemoglobin level (OR: 1.354; $p<0.001$ ) as risk factors associated with $\mathrm{BSI}^{19}$.

In the present study level of education was evaluated as a potentially important risk factor, because patients who recently started undergoing hemodialysis may have had difficulty in performing the necessary maintenance and care of the central venous catheter while at home. However, no significant association between education level and catheter-related infections was observed, this may be because appropriate care of the catheter may be more closely related to the clarity of the instructions provided in hospital, rather than the level of formal education.

Despite the contributions the present study makes in identifying the infection profile of patients with acute kidney injury, a few limitations should be considered. The study was conducted in a single center, in addition, it was conducted in patients with acute kidney injury, affecting the generalizability of the findings. This underscores the importance of developing new studies with the aim of understanding the incidence of and risk factors for these infections in patients with acute kidney injury.

In conclusion, we identified a high incidence of central venous catheter-related infections in patients with acute kidney injury receiving hemodialysis, particularly compared with the incidence in patients with arteriovenous fistula. The occurrence of infections caused by antimicrobial-resistant gram-negative bacteria is noteworthy. Diabetes mellitus as a comorbid condition was the only identified risk factor for infection. It is necessary to improve the care of these devices.

\section{Acknowledgments}

We offer our deepest thanks to the Hospital das Clínicas for providing technical support for the development and implementation of this study.

\section{Conflict of interest}

The authors declare that there is no conflict of interest.

\section{REFERÊNCIAS}

1. Sociedade Brasileira de Nefrologia. Censo da Sociedade Brasileira de Nefrologia de 2014. Available from: http://www.censo-sbn.org.br

2. United States Renal Data System. (USRDS). USRDS 2013. Annual Data Report: Atlas of Chronic Kidney Disease and End-Stage Renal Disease in the United States, National Institutes of Health, National Institute of Diabetes and Digestive and Kidney Diseases. Volume 2, Bethesda: USRDS; 2013. Available from: http://www.usrds.org/atlas.aspx

3. Lukowsky LR, Kheifets L, Arah OA, Nissenson AR, Kalantar-Zadeh K. Patterns and predictors of early mortality in incident hemodialysis patients: new insights. Am J Nephrol. 2012;35(6):548-58.

4. Moysés Neto M, Vieira-Neto OM, Figueiredo JFC. Bacterial colonization in a hemodialysis dual lumen temporary catheter. Rev Soc Bras Med Trop. 2003;36(3):431-2.
5. Bradbury BD, Fissell RB, Albert JM, Anthony MS, Critchlow $\mathrm{CW}$, Pisoni RL, et al. Predictors of early mortality among incident US hemodialysis patients in the Dialysis Outcomes and Practice Patterns Study (DOPPS). Clin J Am Soc Nephrol. 2007;2(1): 89-99.

6. Astor BC, Eustace JA, Powe NR, Klag MJ, Fink NE, Coresh J. Type of vascular access and survival among incident hemodialysis patients: the Choices for Healthy Outcomes in Caring for ESRD (CHOICE) Study. J Am Soc Nephrol. 2005;16(5):1449-55.

7. Dhingra RK, Young EW, Hulbert-Shearon TE, Leavey SF, Port FK. Type of vascular access and mortality in U.S. hemodialysis patients. Kidney Int. 2001;60(4):1443-51.

8. Ishani A, Collins AJ, Herzog CA, Foley RN. Septicemia, access and cardiovascular disease in dialysis patients: the USRDS Wave 2 study. Kidney Int. 2005;68(1):311-8.

9. De Cicco M, Campisi C, Matovic M. Central venous catheter related bloodstream infections: Pathogenesis factors, new perspectives in prevention and early diagnosis. J Vasc Access. 2003;4(3):83-91.

10. O'Grady NP, Alexander M, Burns LA, Dellinger EP, Garland J, Heard SO, et al. Guidelines for the Prevention of Intravascular Catheter-Related Infections. Clin Infect Dis. 2011;52(9):e-162-93.

11. Mesiano ERAB, Merchan-Hamann E. Bloodstream infections among patients using central venous catheters in intensive care units. Rev Lat Am Enfermagem. 2007;15(3):453-9.

12. Arduíno MJ, Tokars JI. Why is an infection control program needed in the hemodialysis setting? Nephrol News Issues. 2005;19(7):44-9.

13. Polkinghorne KR, McDonald SP, Atkins RC, Kerr PG. Vascular access and all-cause mortality: A propensity score analysis. J Am Soc Nephrol. 2004;15(2):477-86.

14. Allon M, Depner TA, Radeva M, Bailey J, Beddhu S, Butterly D, et al. Impact of dialysis dose and membrane on infection-related hospitalization and death: results of the HEMO Study. J Am Soc Nephrol. 2003;14(7):1863-70.

15. Gnass M, Gielish $\mathrm{C}$, Acosta-Gnass S. Incidence of nosocomial hemodialysis-associated bloodstream infections at a county teaching hospital. Am J Infect Control. 2014;42(2):182-4.

16. Colville LA, Lee AH. Retrospective analysis of catheter-related infections in a hemodialysis Unit. Infect Control Hosp Epidemiol. 2006;27(9):969-73.

17. Taylor G, Gravel D, Johnston L, Embil J, Holton D, Paton S. Prospective surveillance for primary bloodstream infections occurring in Canadian hemodialysis units. Infect Control Hosp Epidemiol. 2002;23(12):716-20.

18. Dopirak M, Hill C, Oleksiw M, Dumigan D, Arvai J, English E, et al. Surveillance of hemodialysis-associated primary bloodstream infections: the experience of ten hospital based centers. Infect Control Hosp Epidemiol. 2002;23:721-24.

19. Fysaraki M, Samonis G, Valachis A, Daphnis E, Karageorgopoulos DE, Falagas ME. Incidence, clinical, microbiological features and outcome of bloodstream infections in patients undergoing hemodialysis. Int J Med Sci. 2013;10(12):1632-8.

20. Deshpande LM, Fritsche TR, Moet GJ, Biedenbach DJ, Jones RN. Antimicrobial resistance and molecular epidemiology of vancomycin resistant enterococci from North America and Europe: a report from the SENTRY antimicrobial surveillance pro Gram. Diagn Microbiol Infect Dis. 2007;58(2):163-70.

21. Esmanhoto CG, Taminato M, Fram DS, Belasco AGS, Barbosa DA. Microorganisms isolated from patients on hemodialysis by central venous catheter and related clinical evolution. Acta Paul Enferm. 2013;26(5):413-20. 